\title{
The JUROGAM 3 spectrometer
}

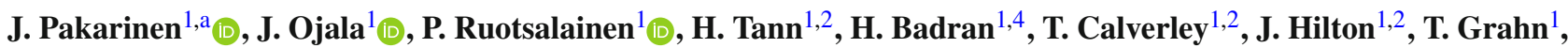 \\ P. T. Greenlees ${ }^{1}$, M. Hytönen ${ }^{1}$, A. Illana ${ }^{1}$, A. Kauppinen ${ }^{1}$, M. Luoma ${ }^{1,3}$, P. Papadakis ${ }^{1,5}$, J. Partanen ${ }^{1}$, K. Porras ${ }^{1}$, \\ M. Puskala ${ }^{1}$, P. Rahkila ${ }^{1}$, K. Ranttila ${ }^{1}$, J. Sarén ${ }^{1}$, M. Sandzelius ${ }^{1}$, S. Szwec ${ }^{1}$, J. Tuunanen ${ }^{1}$, J. Uusitalo ${ }^{1}$, G. Zimba ${ }^{1}$ \\ ${ }^{1}$ Department of Physics, University of Jyvaskyla, P.O. Box 35, 40014 Jyvaskyla, Finland \\ 2 Department of Physics, Oliver Lodge Laboratory, University of Liverpool, P.O. Box 147, Liverpool L69 7ZE, UK \\ ${ }^{3}$ Helsinki Institute of Physics, University of Helsinki, P.O. Box 64, 00014 Helsinki, Finland \\ ${ }^{4}$ Present Address: STUK-Radiation and Nuclear Safety Authority, P.O. Box 14, 00881 Helsinki, Finland \\ ${ }^{5}$ Present Address: STFC Daresbury Laboratory, Daresbury, Warrington WA4 4AD, UK
}

Received: 20 December 2019 / Accepted: 9 April 2020 / Published online: 25 May 2020

(C) The Author(s) 2020

Communicated by Navin Alahari

\begin{abstract}
The JUROGAM 3 spectrometer has been constructed for in-beam $\gamma$-ray spectroscopy experiments in the Accelerator Laboratory of the University of Jyväskylä, Finland. JUROGAM 3 consists of germanium-detector modules in a compact geometry surrounding a target to measure $\gamma$ rays emitted from radioactive nuclei. JUROGAM 3 can be employed in conjunction with one of two recoil separators, the MARA vacuum-mode separator or the RITU gas-filled separator, and other ancillary devices.
\end{abstract}

\section{Introduction}

Since 2003 the two incarnations of JUROGAM germaniumdetector arrays have provided a great wealth of in-beam spectroscopic data in mainly recoil-decay tagging experiments at the RITU gas-filled separator [1] in the Accelerator Laboratory of the University of Jyväskylä, Finland (JYFL). The driving scientific themes and objectives of the JUROGAM campaigns can be summarised as follows:

- Providing solid configuration assignments in odd-mass transfermium nuclei.

- Investigating shape coexistence in the neutron-deficient lead region.

- Studying the structure of nuclei close to the proton dripline.

In order to better address our physics program for nuclei located around the $N=Z$ line and at the proton drip line,

J. Partanen: Deceased.

a e-mail: janne.pakarinen@jyu.fi (corresponding author) a new vacuum-mode separator MARA has been developed $[2,3]$. MARA allows for the separation of reaction products from the primary beam in symmetric and inverse kinematics fusion-evaporation reactions. It has proven to be an excellent tool for decay spectroscopy of very proton-rich nuclei. To date, nuclei ranging from ${ }^{45} \mathrm{Cr}$ to ${ }^{170} \mathrm{Hg}$ have been successfully studied, including the discovery of five new isotopes [4-6].

The JUROGAM 3 spectrometer carries the same detector configuration as its predecessor JUROGAM II. The main difference between the two spectrometers is that the JUROGAM 3 array can be swiftly moved between the RITU and MARA separators. In practise, the novel concept allows for back-to-back in-beam $\gamma$-ray spectroscopy experiments to be performed at the MARA and RITU separators. The advent of the JUROGAM 3 spectrometer in conjunction with MARA has raised sensitivity to probe exotic nuclei around the $N=Z$ line employing inbeam spectroscopy to a new level. The physics questions to be addressed include topics such as isospin symmetry breaking [7], neutron-proton pairing [8] and shape coexistence [9]. In this paper, key aspects and performance of the JUROGAM 3 spectrometer will be presented. Essential properties, operational aspects and outcome of the three JUROGAM spectrometers are compared in Table 1.

\section{Description of JUROgam 3}

The JUROGAM 3 germanium-detector array inherits characteristics from the EUROGAM II array [10]. EUROGAM II consisted of two types of germanium detectors: tapered singlecrystal Phase 1 detectors [11] and composite Clover detectors [12]. The latter houses four individual germanium crystals 
Table 1 Key properties, operation and outcome of the JUROGAM arrays

\begin{tabular}{|c|c|c|c|}
\hline & JUROGAM I & JUROGAM II & JUROGAM 3 \\
\hline Detector & 43 tapered $^{\mathrm{a}}$ & 15 tapered & 15 tapered \\
\hline configuration & & 24 Clover $^{\mathrm{b}}$ & 24 Clover \\
\hline Efficiency ${ }^{\mathrm{c}}$ & $4.3 \%$ & $5.2 \%$ & $5.2 \%$ \\
\hline Operating years & 2003-2008 & 2008-2017 & $2019-$ \\
\hline Experiments $^{\mathrm{d}}$ & 61 & 81 & 7 \\
\hline Beamtime hours & $13,700+$ & $19,600+$ & $2100+$ \\
\hline Publications ${ }^{\mathrm{e}}$ & 75 & 71 & - \\
\hline
\end{tabular}

${ }^{\text {a }}$ Single-crystal EUROGAM Phase1 and GASP type detectors

${ }^{\mathrm{b}}$ Segmented EUROGAM II Clover detectors

${ }^{c}$ For the detection of $1332 \mathrm{keV} \gamma$ rays

${ }^{\mathrm{d}}$ Excluding commissionings, tests and reruns of experiments

${ }^{\mathrm{e}}$ In peer-reviewed journals excluding conference proceedings within the same cryostat. Detailed description and performance of these germanium detectors, various $\gamma$-ray spectrometers and the development of European germaniumdetector arrays can be found in references [10-15] and references therein.

Each germanium detector in the JUROGAM 3 array is accompanied with a bismuth germanate (BGO) Comptonsuppression shield and a heavy-metal collimator [11,12]. The BGO shields allow $\gamma$ rays that deposit only part of their energy in the germanium crystal to be rejected from the data (vetoing), while the heavy-metal collimators prevent direct hits of $\gamma$ rays emitted from the target in the BGO shields. A germanium detector, related BGO shield and heavy-metal collimator are commonly called as a detector module below.

The detector modules are mounted so that the reaction target is located in the focus of the array. The target positions of the RITU and MARA recoil separators are well defined and can not be moved more than $\sim 10 \mathrm{~cm}$ upstream without losses in separator acceptance. Therefore, in order to fit the EUROGAM II-type array with these recoil separators, the two detector rings located downstream from the target in the original EUROGAM II array have been removed. As a result, the JUROGAM 3 array consists of four detector rings as illustrated in Fig. 1. Five detector modules with tapered Phase1or GASP-type detectors are mounted in a ring at $157.6^{\circ}$ and another ten in a ring at $133.6^{\circ}$ with respect to the beam axis. The detector modules with Clover detectors are mounted in two rings at $104.5^{\circ}$ and $75.5^{\circ}$ with respect to the beam axis, each ring housing 12 detector modules. Consequently, the full array consists of 39 detector modules and 111 individual germanium crystals.

In addition to recoil separators, JUROGAM 3 can be combined with various other ancillary devices. In particular, the JYTube (Jyväskylä-York Tube) charged-particle veto detector [17] surrounding the JUROGAM 3 target brings added sensitivity to probe neutron-evaporation channels, while the SAGE spectrometer can be employed in simultaneous in-beam

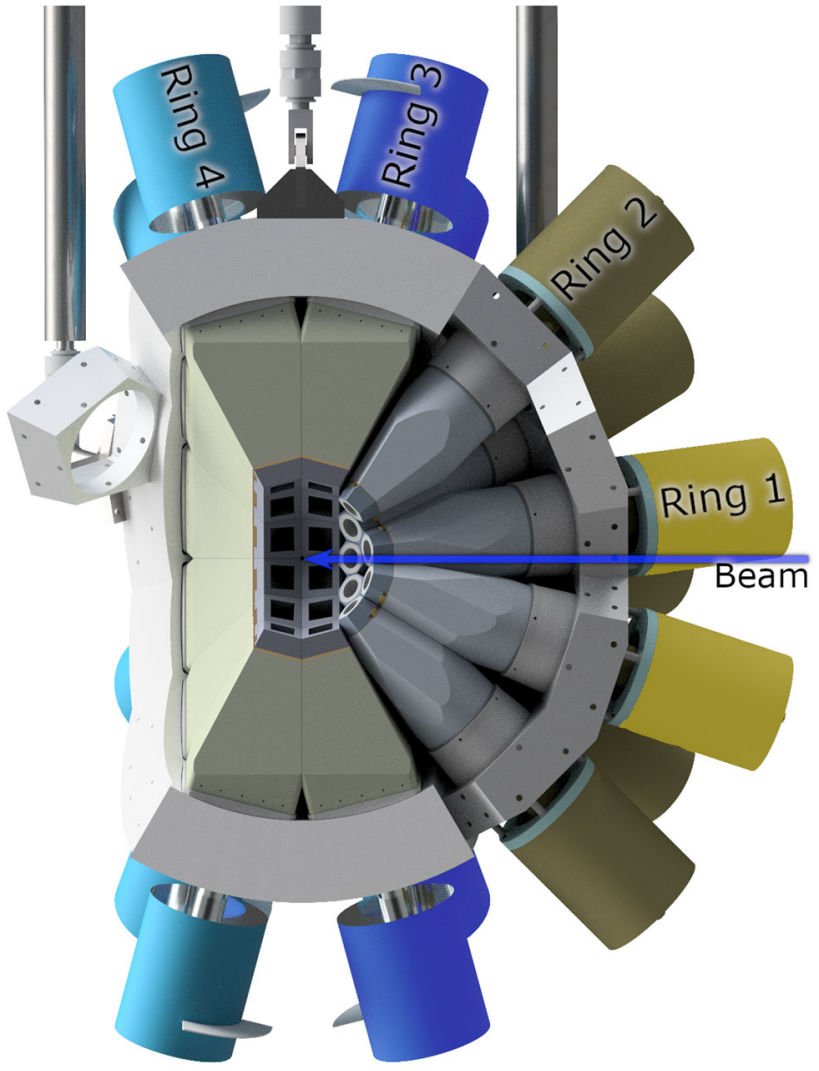

Fig. 1 Rendered design drawing of the JUROGAM 3 germaniumdetector array. One hemisphere, supporting frame, beam line, target chamber and separators have been removed for visualisation purposes. Different detector rings have been labelled and marked with different colours. The heavy-ion beam delivered by the K130 cyclotron enters from the right and is marked with an arrow pointing to the target position

$\gamma$-ray and conversion electron experiments [18]. Lifetimes of states de-exciting via $\gamma$-ray emission can be measured using a plunger device, such as DPUNS [19].

As the majority of experiments at RITU and MARA employ fusion-evaporation reactions, the germanium detectors mea- 


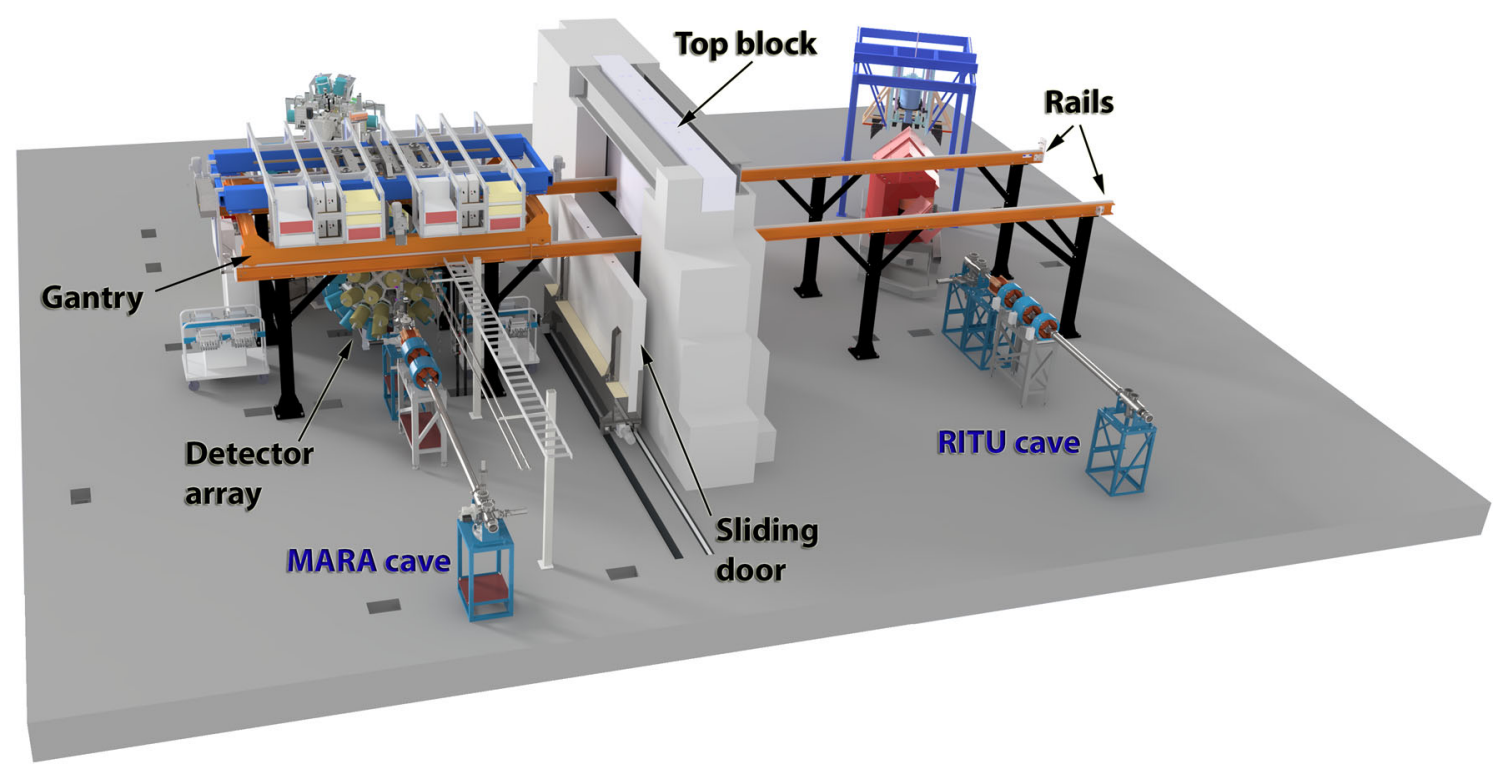

Fig. 2 Rendered design drawing of the JUROGAM 3 transport system with the germanium-detector array at the target position of MARA. Essential parts have been labeled, the roof and walls of experimental caves have been partially removed for visualisation purposes

suring prompt $\gamma$ rays are also exposed to neutrons that can dislocate atoms in the germanium crystal lattice. This radiation damage causes incomplete charge collection and reduces the performance of a detector which manifests itself as a tail at the low-energy side of the photopeak in the $\gamma$-ray energy spectrum. In practise, the effect can go unnoticed until the bias voltage is removed from a detector. Although the damage can be mitigated by annealing the detector, it is of great importance to have the detectors biased through-out the experimental campaign as annealing of the full array of detectors would require a considerably long break between experiments. Therefore, the JUROGAM 3 spectrometer was designed to allow for transportation between the adjacent RITU and MARA separators without the need to remove the bias voltage and to warm up detectors. A rendered design drawing of the JUROGAM 3 transport system in the experimental cave of the RITU and MARA separators is shown in Fig. 2. Key aspects and solutions to facilitate transportation between the two separators are discussed in more details below.

\subsection{The JUROGAM 3 transporter}

The JUROGAM 3 array is supported with six vertical beams from the transportable gantry. The array consists of two hemispheres which can be individually opened allowing unimpeded access to the target area. The gantry lies on rails and in addition to the germanium-detector array it also houses:

- preamplifier power supplies for the germanium detectors and associated BGO shields;
- high-voltage (HV) power supplies to provide bias voltage for the germanium detectors and power to the photomultiplier tubes of the BGO shields;

- control system for automatic liquid nitrogen $\left(\mathrm{LN}_{2}\right)$ filling of the germanium-detector Dewars;

- all necessary cables for detector signals, preamplifier power, HV, temperature readout, bias shutdown;

- single-ended to differential (SoD) converter cards for detector signals (see Sect. 2.2),

- motors to move the gantry and hemispheres; and

- three different types of electric power distributions.

The gantry can be moved up to $60 \mathrm{~cm}$ upstream of the target position in the beam-line direction and $9 \mathrm{~m}$ perpendicular to the beam-line direction. The former allows for better matching to the geometrical requirements of ancillary detectors, while the latter is exploited when moving the spectrometer between the target positions of the two separators. Transportation can be made without power interruption i.e. whilst keeping the germanium detectors biased and cold. Typically, a move lasts for $2-3 \mathrm{~h}$, which is much shorter than the typical 8-hour $\mathrm{LN}_{2}$ filling cycle. Therefore, during transportation the $\mathrm{LN}_{2}$ supply hoses can be disconnected from the detectors. The $\mathrm{LN}_{2}$ manifolds and purge containers are installed on two mobile trolleys which are directly connected to the main 7.1-ton $\mathrm{LN}_{2}$ tank, removing the need for buffer Dewars close to the array. In order to comply with radiation-safety regulations and to allow for work in one cave while running an experiment in the adjacent cave, the door between caves can be closed with sliding and liftable concrete blocks. 
The JUROGAM 3 spectrometer employs electricity from three different sources which can be classified as follows:

1. general electricity ("dirty") used for the transporter motors, $\mathrm{LN}_{2}$ solenoid valves, working lights etc;

2. Uninterrupted Power Supply (UPS) ("clean") for detector $\mathrm{HV}$, germanium-detector preamplifiers, automatic $\mathrm{LN}_{2}$ filling system control; and

3. "clean" measurement electricity for the BGO preamplifliers and SoD converter cards.

The "clean" UPS and measurement electricity supplies share the same grounding point, but are separated from the general electricity ground with an isolation transformer, reducing noise pick-up introduced by numerous power supplies employed in the laboratory.

\subsection{Detector signal chain and data acquisition}

The single-ended signals from the germanium-detector preamplifiers are converted to differential signals using JYFL-designed 16-channel SoD converter cards. This allows for the use of twisted-pair ribbon cabling and provides an easy-to-handle option to transport the signals from the measurement cave over $30 \mathrm{~m}$ to the data acquisition (DAQ) room without compromising the signal quality. Here conversion from differential to single-ended signalling required by the digitisers is done with 16-channel differential to single-ended (DoS) cards, also of JYFL design. The signals are then digitised by Lyrtech/Nutaq VHS-ADC with 14-bit accuracy and $100 \mathrm{MHz}$ sampling rate. For each suppression shield, all photomultiplier outputs are daisy-chained and fed into digitisers using a similar signal chain as described above for the germanium detectors. Signal chain for a Clover detector module is schematically shown in Fig. 3.

Signal amplitudes from the germanium detectors are extracted from the digitised signals using a Moving Window Deconvolution (MWD) algorithm [22] running real-time in a FPGA chip on each digitiser module. Each interaction in the detector is time-stamped using an external $100 \mathrm{MHz}$ clock distributed across all modules in the DAQ system. The BGO shield energy signals are not read out directly. Instead, the germanium data word is marked if the suppression shield has fired within a preset coincidence window of typically

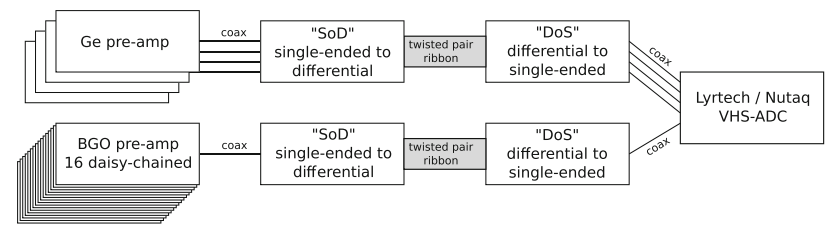

Fig. 3 Signal chain for one Clover detector module. Three Clover detectors can be instrumented using one VHS-ADC
Table 2 Typical MWD parameters applied for a JUROGAM 3 germanium-detector channel

\begin{tabular}{|c|c|}
\hline CFD threshold ${ }^{\mathrm{a}}$ & $5-25$ \\
\hline TFA shaping time ${ }^{b}$ & $120 \mathrm{~ns}$ \\
\hline Shaping time & $4.0 \mu \mathrm{s}$ \\
\hline Rise time & $2.5 \mu \mathrm{s}$ \\
\hline Peak sample & $3.8 \mu \mathrm{s}$ \\
\hline Peak separation & $7 \mu \mathrm{s}$ \\
\hline Decay time constant & $45-55 \mu \mathrm{s}$ \\
\hline Baseline average $^{\mathrm{c}}$ & 4 \\
\hline Baseline update $^{\mathrm{d}}$ & $2 \mu \mathrm{s}$ \\
\hline Preset coincidence window (BGO veto) & $1200 \mathrm{~ns}$ \\
\hline
\end{tabular}

${ }^{\text {a }}$ Corresponds to the low-energy threshold of $\sim 10-30 \mathrm{keV}$

b Timing Filter Amplifier (TFA)

${ }^{c} 2^{4}=16$ samples to define baseline

${ }^{\mathrm{d}}$ Sample interval before triggered signal

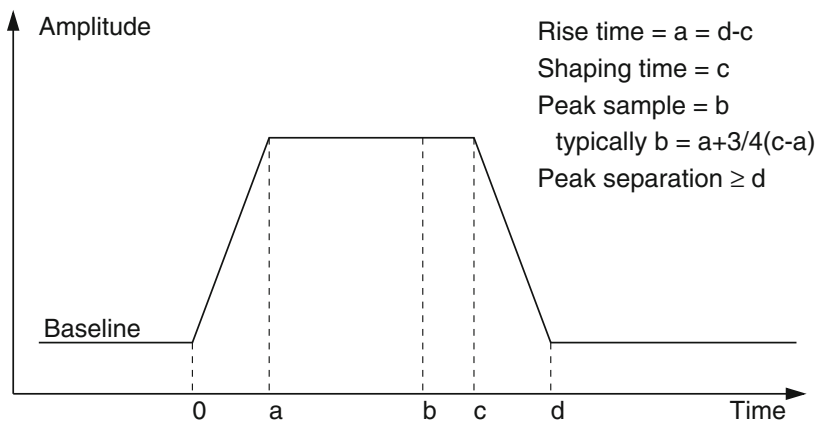

Fig. 4 Relation of the MWD parameters to the trapezoidal filter employed in the TDR DAQ system

$600 \mathrm{~ns}$ in width, using the timestamped information from the Constant-Fraction Discriminator (CFD) algorithm running in the FPGA chip.

The DAQ system, including that of the focal plane and ancillary detectors is based on the concept of Total Data Readout as described in Ref. [23]. Typical MWD filter parameters for a germanium-detector channel are listed in Table 2 and their relation to the trapezoidal filter is explained in Fig. 4.

\section{Performance}

3.1 Detector resolutions and peak-to-total values

Offline measurements were conducted using ${ }^{60} \mathrm{Co},{ }^{133} \mathrm{Ba}$ and ${ }^{152} \mathrm{Eu}$ calibration sources that were installed on the target fan inside the JUROGAM 3 target chamber and data were collected employing the DAQ system described in Sect. 2.2. The full-width at half maximum (FWHM) resolution have been determined at two different energies, namely at $356 \mathrm{keV}$ and $1332 \mathrm{keV}$ for all individual germanium crystals. A Clover 
Table 3 Average FWHM resolution values of the JUROGAM 3 detectors with standard deviations measured in the array and in the test set-up

\begin{tabular}{lll}
\hline & \multicolumn{2}{l}{ FWHM resolution at } \\
\cline { 2 - 3 } $356 \mathrm{keV}[\mathrm{keV}]$ & $1332 \mathrm{keV}[\mathrm{keV}]$ \\
\hline JUROGAM 3 array & & \\
Tapered detectors & $2.59(59)$ & $3.20(55)$ \\
Clover detectors $^{\mathrm{a}}$ & $2.06(34)$ & $2.80(32)$ \\
Clover detectors $^{\mathrm{b}}$ & $2.29(25)$ & $3.27(33)$ \\
Full array $^{\mathrm{a}}$ & $2.11(41)$ & $2.85(37)$ \\
Full array $^{\mathrm{b}}$ & $2.38(41)$ & $3.25(40)$ \\
Test set-up & & $2.88(37)$ \\
Tapered detectors $_{\text {Clover detectors }}^{\mathrm{a}}$ & - & $2.67(22)$ \\
\hline
\end{tabular}

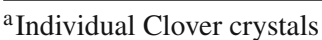

${ }^{\mathrm{b}}$ Employing add-back for Clover detectors

detector can be operated in direct detection mode, where each crystal is used as a single detector, or in so-called addback mode. In the latter, events recorded in adjacent crystals (including diagonal crystals) within $200 \mathrm{~ns}$ temporal coincidence window were considered as Compton-scattered $\gamma$ rays and their energies were summed together. While the add-back mode improves the photopeak detection efficiency and peak-to-total value (see below), it slightly deteriorates the FWHM resolution value. In Table 3, average FWHM resolution values have been listed for tapered and Clover detectors and for the full array. For comparison, the average FWHM resolution values at $1332 \mathrm{keV}$ measured for tapered and Clover detectors in test set-up have also been presented in Table 3. The test set-up was in an electromagnetically shielded room (commercial Euroshield) and employed conventional NIM-based electronics. Each detector was tested with a ${ }^{60} \mathrm{Co}$ source placed at $25 \mathrm{~cm}$ distance in front of the detector and data was collected using $2 \mu$ s shaping time in the linear amplifier.

The FWHM resolution and suppression of escaped $\gamma$ rays for tapered and Clover detector modules are demonstrated in $\gamma$-ray energy spectra obtained with ${ }^{60} \mathrm{Co}$ source in Fig. 5. The unsuppressed singles $\gamma$-ray energy spectra are quoted as "total". In the spectra labelled as "suppressed", $\gamma$ rays in coincidence with events in the corresponding BGO shield were removed from data. The spectra for removed $\gamma$ rays are labelled as "rejected". In the top panel, singles $\gamma$-ray energy spectra obtained with a Clover detector operated in add-back mode is shown. An average add-back gain of 1.49 (2) at $1332 \mathrm{keV}$ has been extracted, which is well in line with the corresponding value reported earlier [12]. The bottom panel presents the same for a tapered detector. It is clear how the performance of both types of detector modules benefit from the BGO veto. This can be expressed quantitatively with a peak-to-total value, that is defined as the

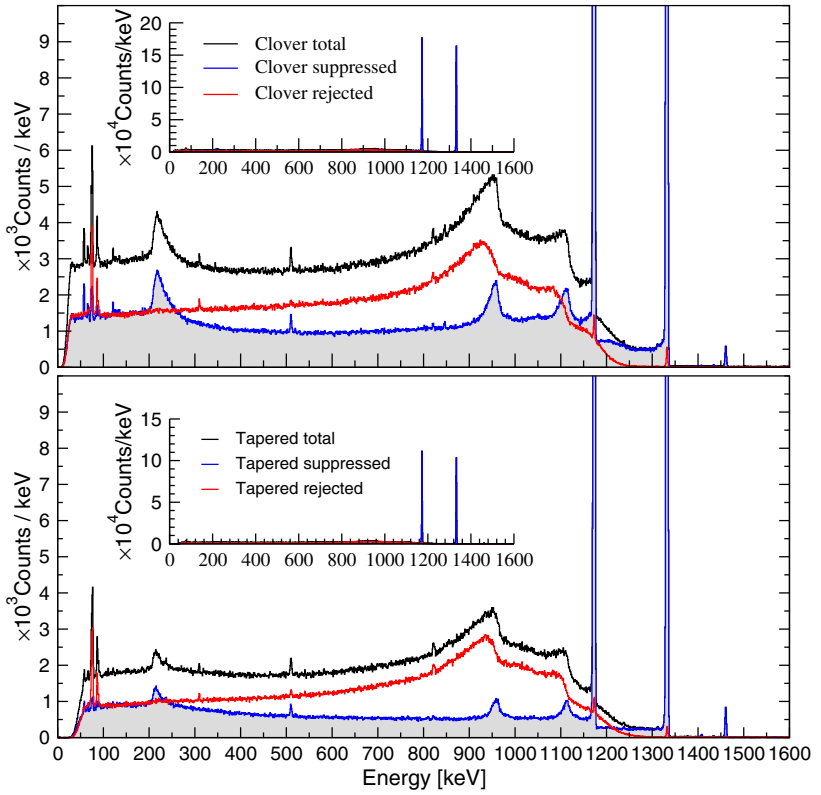

Fig. 5 Singles $\gamma$-ray energy spectra obtained using a ${ }^{60}$ Co calibration source with a Clover detector module in add-back mode (top) and a tapered detector module (bottom) in the JUROGAM 3 array employing three different conditions: (1) total $\gamma$-ray energy (black), (2) $\gamma$-ray energy vetoed with BGO shield (blue), 3) $\gamma$-ray energy triggered with BGO shield (red). Insets show the same spectra with full scale $y$-axis

Table 4 Performance of the JUROGAM 3 array with and without the JYTube detector. Peak-to-total values are listed for the full array and different detector types. $\gamma$-ray photopeak detection efficiency is given at two different energies

\begin{tabular}{|c|c|c|}
\hline Property & JUROGAM 3 & $\begin{array}{l}\text { JUROGAM } 3 \\
+ \text { JYTube }\end{array}$ \\
\hline \multicolumn{3}{|l|}{ Peak-to-total } \\
\hline Full array & $0.47(1)$ & $0.44(1)$ \\
\hline Clover detectors & $0.47(1)$ & $0.43(1)$ \\
\hline Tapered detectors & $0.47(1)$ & $0.45(1)$ \\
\hline \multicolumn{3}{|l|}{$\gamma$-ray efficiency [\%] } \\
\hline At $356 \mathrm{keV}$ & $11.3(2)$ & $9.8(1)$ \\
\hline At $1332 \mathrm{keV}$ & $5.2(1)$ & $4.1(1)$ \\
\hline
\end{tabular}

ratio between the sum of the photopeak areas divided by the total number of counts in the spectrum for energies ranging from 100 to $1350 \mathrm{keV}$. Peak-to-total values obtained for the full JUROGAM 3 array, Clover and tapered detectors with and without the JYTube detector are given in Table 4.

\subsection{Detection efficiency}

The photopeak detection efficiency of the JUROGAM 3 array as a function of $\gamma$-ray energy was extracted employing ${ }^{133} \mathrm{Ba}$ and ${ }^{152} \mathrm{Eu}$ sources that provide data points up to $1408 \mathrm{keV}$. Data points were fitted to the following functional: 


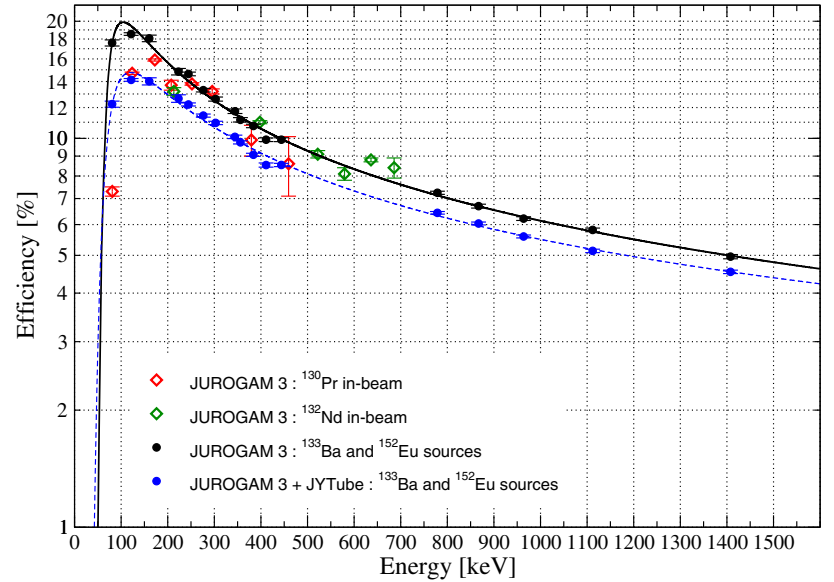

Fig. $6 \gamma$-ray detection efficiency of JUROGAM 3. Filled circles represent efficiencies determined with ${ }^{133} \mathrm{Ba}$ and ${ }^{152} \mathrm{Eu}$ calibration sources for the JUROGAM 3 array (black) and the JUROGAM 3 array combined with the JYTube detector (blue). Continuous and dashed lines are fit to the source data (corresponding colours). Open diamonds represent detection efficiency extracted from in-beam data obtained for ${ }^{130} \mathrm{Pr}$ (red) and ${ }^{132} \mathrm{Nd}$ (green) nuclei

$\epsilon=\exp \left(\left(\left(a+b x+c x^{2}\right)^{-g}+\left(d+e y+f y^{2}\right)^{-g}\right)^{-\frac{1}{g}}\right)$,

where $x=\ln \left(E_{\gamma} / 100 \mathrm{keV}\right)$ and $y=\ln \left(E_{\gamma} / 1000 \mathrm{keV}\right)$. The relative efficiency curve was normalised with the absolute $\gamma$-ray efficiency determined from ${ }^{60} \mathrm{Co}$ data employing the sum-peak method $[20,21]$ and verified with the known activity of the source. The efficiency curve (with and without JYTube) together with data points are shown in Fig. 6. In addition, detection efficiency extracted from in-beam data obtained for ${ }^{130} \mathrm{Pr}$ and ${ }^{132} \mathrm{Nd}$ nuclei is also plotted (see Sect. 3.3). The efficiency of JUROGAM 3 detectors at $356 \mathrm{keV}$ and at $1332 \mathrm{keV}$, with and without the JYTube detector, is given in Table 4.

\subsection{In-beam performance}

The first JUROGAM 3 experiment was dedicated to the search for highly-deformed proton emitters with the MARA separator. A beam of ${ }^{78} \mathrm{Kr}$ ions impinged on a $0.75-\mathrm{mg} / \mathrm{cm}^{2}$ thick ${ }^{58} \mathrm{Ni}$ target. The beam energy was chosen to be $365 \mathrm{MeV}$ and the beam intensity employed was up to $3 \mathrm{pnA}$. The set-up was optimised to study ${ }^{131} \mathrm{Eu}$ produced in the $\mathrm{p}-4 \mathrm{n}$ evaporation channel. Fusion-evaporation residues were separated from primary and scattered beams with MARA. In this experiment, the reference particle mass was set to $A=129$ and MARA was operated with mass slits open. This allowed for five different charge states with mass-to-charge ratio ranging from 3.5 to 4.0 to be transported to the focal plane and as a result, the MARA transmission efficiency is estimated to be higher than $50 \%$. At the MARA focal plane, recoils were implanted
Table 5 Typical rates recorded during the ${ }^{58} \mathrm{Ni}\left({ }^{78} \mathrm{Kr}, \mathrm{p} 4 \mathrm{n}\right){ }^{131}$ Eu experiment

Beam intensity

$3.0 \mathrm{pnA}$

Tapered detector count rate

$7.5 \mathrm{kHz}$

Clover detector count rate (one crystal)

$5.0 \mathrm{kHz}$

Focal plane MWPC detector count rate

$8.5 \mathrm{kHz}$

Focal plane implantation detector rate

$5.9 \mathrm{kHz}$

Data transfer to storage

$2400 \mathrm{kB} / \mathrm{s}$

into a $300 \mu \mathrm{m}$-thick BB20-type position sensitive doublesided silicon strip detector to allow for spatial and temporal correlation of recoils and their subsequent decay. A transmission multiwire proportional counter (MWPC) upstream of the implantation detector was employed to obtain energy loss and time-of-flight information for the recoils. Outside the focal-plane vacuum chamber, an array of four EUROGAM II Clover detectors was set-up in tight geometry for measuring $\gamma$ rays with detection efficiency of $\sim 12 \%$ at $124 \mathrm{keV}$. Recoil velocity in this experiment was $5.7 \%$ of the speed of light, thus transmission time through the MARA separator was $\sim 400 \mathrm{~ns}$. Typical count rates recorded during the experiment are given in Table 5.

In a typical JUROGAM 3 experiment, $\gamma$ rays are emitted in flight from nuclei moving at velocities in the range of 1-8\% of the speed of light. This gives rise to the Doppler broadening of $\gamma$-ray peaks in the energy spectrum, which can be expressed as

$\Delta E=\frac{v}{c} \sin \theta \Delta \theta E$

where $\Delta \theta$ is the opening angle of the detector, $\theta$ is the detector angle with respect to the recoil velocity vector ( $\sim$ beam axis), $v$ is the recoil velocity, $c$ is the speed of light and $E$ is the $\gamma$-ray energy. For example, assuming a recoil velocity of $v=$ $0.057 c$, an average Doppler broadening for a Clover crystal at $\theta=75.5^{\circ}$ with $\Delta \theta=6.5^{\circ}$ is $0.62 \%$ of the $\gamma$-ray energy, i.e. $3.1 \mathrm{keV}$ for a peak at $500 \mathrm{keV}$. The effect of Doppler broadening is demonstrated in Fig. 7, where the FWHM resolution values obtained with ${ }^{133} \mathrm{Ba}$ and ${ }^{152} \mathrm{Eu}$ calibration sources are compared to values extracted for transitions in the ${ }^{131} \mathrm{Nd}$ and ${ }^{132} \mathrm{Nd}$ nuclei obtained in the in-beam experiment presented above.

In order to avoid a high X-ray flux arising from the beam impinging on the target (typical in heavy-element fusionevaporation experiments), two layers of absorbers, namely $0.23-0.26 \mathrm{~mm}$ of $\mathrm{Sn}$ and $0.50-0.64 \mathrm{~mm}$ of $\mathrm{Cu}$ were installed in front of the germanium detectors. As presented in Table 5 , the individual germanium detector count rates were well below $10 \mathrm{kHz}$. While the absorbers introduce physical lowenergy threshold for detection of $\gamma$ rays, they also reduce the detection efficiency at low energies. The in-beam detection 


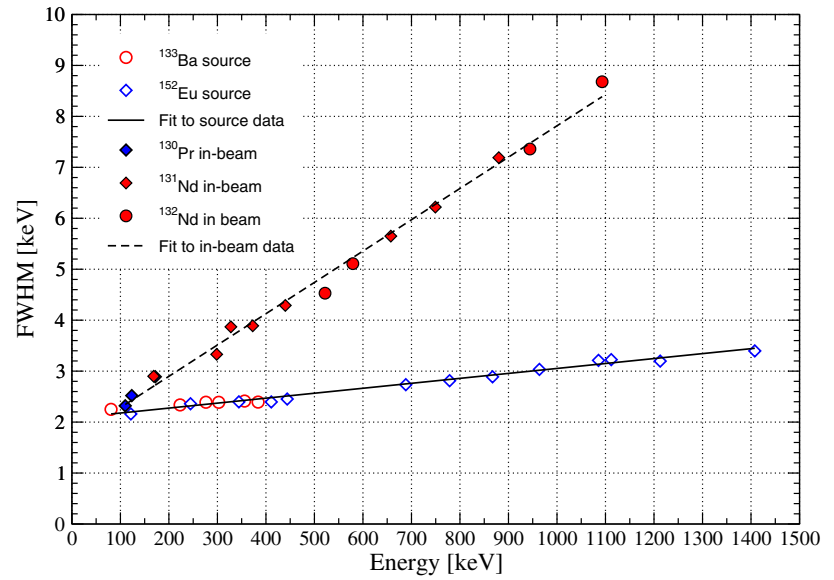

Fig. 7 FWHM resolution values obtained for the JUROGAM 3 array plotted as a function $\gamma$-ray energy. $\gamma$ rays originating from ${ }^{133} \mathrm{Ba}$ and ${ }^{152} \mathrm{Eu}$ calibration sources are labelled with filled symbols. $\gamma$ rays assigned to ${ }^{130} \mathrm{Pr},{ }^{131} \mathrm{Nd}$ and ${ }^{132} \mathrm{Nd}$ nuclei (open symbols) were emitted in-flight in the in-beam experiment described in the text. Clover detectors were operated in add-back mode

efficiency of JUROGAM 3 (see Fig. 6) was extracted exploiting $\gamma-\gamma$ coincidence data obtained for ${ }^{130} \mathrm{Pr}$ and ${ }^{132} \mathrm{Nd}$ nuclei. In case of ${ }^{130} \mathrm{Pr}$, subsequent $\gamma$-ray de-excitations in coincidence with the $596 \mathrm{keV}$ transition feeding the $\left(11^{-}\right)$state were used. Normalisation was made with the $296 \mathrm{keV}$ transition of the cascade. In case of ${ }^{132} \mathrm{Nd}$, the $\left(14_{1}^{+}\right) \rightarrow\left(12_{1}^{+}\right)$transition was used as the gate and normalisation was made with the $522 \mathrm{keV}$ $6_{1}^{+} \rightarrow 4_{1}^{+}$transition. Concerning low energies, detection efficiency at $81 \mathrm{keV}$ and $124 \mathrm{keV}$ was determined to be $7.3 \%$ and $15.1 \%$, respectively. As seen in Fig. 6, these are lower than the corresponding values measured with the ${ }^{152} \mathrm{Eu}$ and ${ }^{133} \mathrm{Ba}$ calibration sources. This can be explained by absorbers that were not used in source measurements.

A sample set of in-beam $\gamma$-ray energy spectra, Doppler corrected for fusion-evaporation residues is shown in Fig. 8. The evolution of spectral purity is evident when applying more conditions as demonstrated below.

Panel a) shows prompt $\gamma$-ray energy spectrum, which is dominated by a peak at $455 \mathrm{keV}$ arising from the Coulomb excitation of the $2_{1}^{+}$state in ${ }^{78} \mathrm{Kr}$ that was used as the beam. The $\gamma$-ray energy spectrum in panel b) is gated with recoils detected in the MARA focal-plane implantation detector. In addition to more pronounced peaks associated with ${ }^{128-130} \mathrm{Pr}$ and ${ }^{130-132} \mathrm{Nd}$ nuclei, the peak at $79 \mathrm{keV}$ that was present in panel a) has disappeared suggesting that the corresponding transition is delayed.

Panels c) and d) present recoil-gated prompt $\gamma$-ray energy spectra tagged with delayed $79 \mathrm{keV} \gamma$ rays observed at the MARA focal plane within $1 \mu \mathrm{s}$ after recoil implantation. A cascade of $\gamma$ rays associated with the ${ }^{130} \mathrm{Pr}$ nucleus can be identified [24]. These transitions are even more pronounced in panel d), where recoil-gated, isomer-tagged prompt $\gamma$ rays
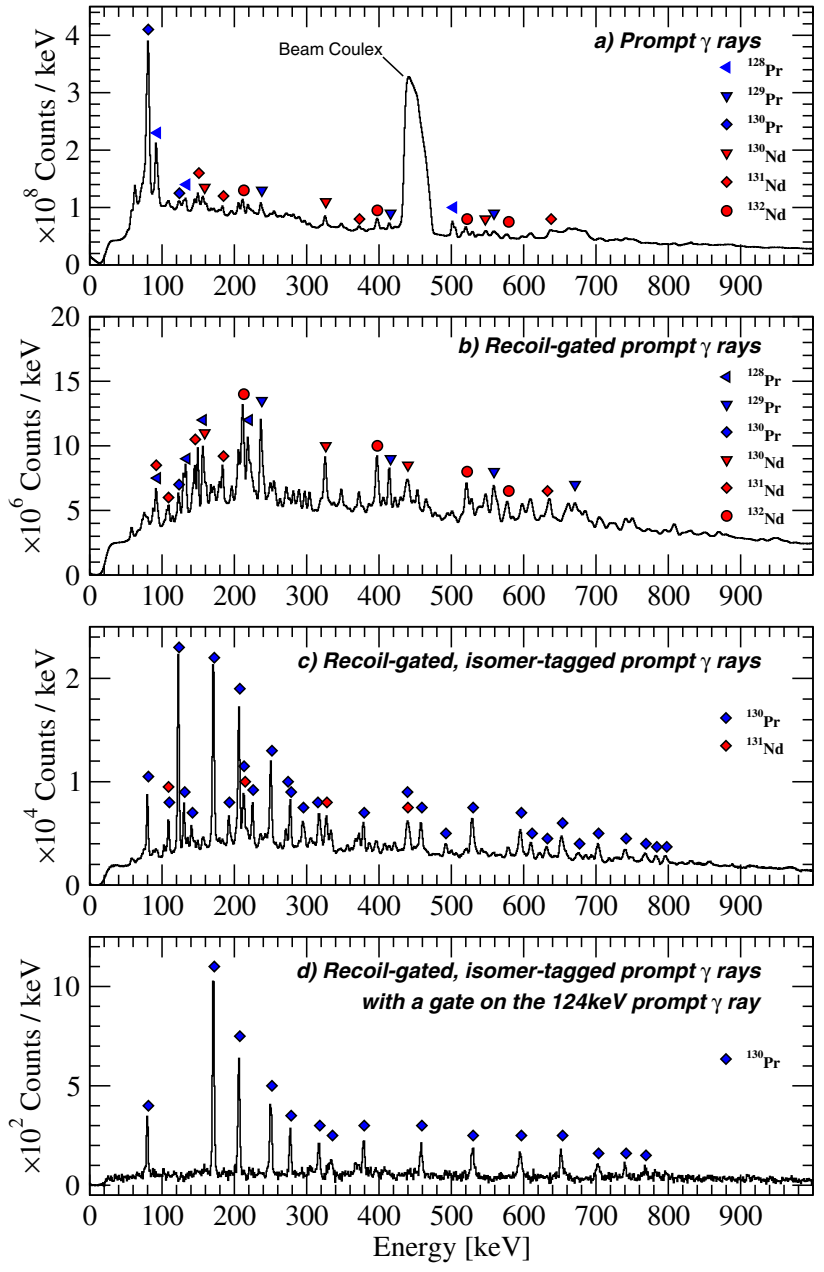

Fig. $8 \gamma$-ray energy spectra obtained with the JUROGAM 3 spectrometer at the MARA separator exploiting ${ }^{78} \mathrm{Kr}+{ }^{58} \mathrm{Ni}$ reaction. In the panel a) prompt $\gamma$-ray energy spectrum is presented. The panel b) shows prompt $\gamma$-ray energy spectrum in coincidence with recoils detected at the MARA focal plane. In the panel c), recoil-gated isomer-tagged prompt $\gamma$-ray energy spectrum is shown and the panel d) presents the same with a coincidence gate on prompt $124 \mathrm{keV} \gamma$ rays. The origin of the most prominent peaks is indicated

in coincidence with the prompt $124 \mathrm{keV} 8_{1}^{-} \rightarrow 7_{1}^{-}$transition in ${ }^{130} \mathrm{Pr}$ are presented. Consequently, the $79 \mathrm{keV}$ transition is considered as a new isomeric state in ${ }^{130} \operatorname{Pr}$ [25].

\section{Summary}

The JUROGAM 3 spectrometer, enabling back-to-back inbeam spectroscopic experiments at the RITU and MARA separators, has been commissioned. In particular, JUROGAM 3 together with MARA allows the investigation of nuclei at the proton dripline at an unprecedented level. The results obtained in the first experimental campaign with MARA are intriguing and exciting - a broad physics program lies ahead. 
Acknowledgements Open access funding provided by University of Jyväskylä (JYU). This work has been supported through the Academy of Finland under funding for the Finnish Research Infrastructures (Contract No. 305272). The European Gamma-Ray Spectroscopy pool is acknowledged.

Data Availability Statement This manuscript has no associated data or the data will not be deposited. [Author's comment: The data of this publication are available from the authors upon request.]

Open Access This article is licensed under a Creative Commons Attribution 4.0 International License, which permits use, sharing, adaptation, distribution and reproduction in any medium or format, as long as you give appropriate credit to the original author(s) and the source, provide a link to the Creative Commons licence, and indicate if changes were made. The images or other third party material in this article are included in the article's Creative Commons licence, unless indicated otherwise in a credit line to the material. If material is not included in the article's Creative Commons licence and your intended use is not permitted by statutory regulation or exceeds the permitted use, you will need to obtain permission directly from the copyright holder. To view a copy of this licence, visit http://creativecomm ons.org/licenses/by/4.0/.
5. A. Briscoe et al., private communication

6. J. Uusitalo et al., private communication

7. M.A. Bentley, S.M. Lenzi, Prog. Part. Nucl. Phys. 59, 497 (2007)

8. B. Cederwall et al., Nature 469, 68 (2011)

9. K. Heyde, J.L. Wood, Rev. Mod. Phys. 83, 1467 (2011)

10. J. Eberth, J. Simpson, Progr. Part. Nucl. Phys. 60, 283 (2008)

11. C. Beausang et al., Nucl. Instrum. Methods A 313, 37 (1992)

12. G. Duchêne et al., Nucl. Instrum. Methods A 432, 90 (1999)

13. F. Beck, Progr. Part. Nucl. Phys. 28, 443 (1992)

14. J. Eberth, Progr. Part. Nucl. Phys. 28, 495 (1992)

15. P. Nolan et al., Annu. Rev. Nucl. Part. Sci. 45, 561 (1994)

16. European Gamma-Ray Spectroscopy pool, http://gammapool. in 2 p3.fr/index.php

17. P. Ruotsalainen et al., private communication

18. J. Pakarinen et al., Eur. Phys. J. A 50, 53 (2014)

19. M.J. Taylor et al., Nucl. Instrum. Methods A 707, 143 (2013)

20. G.A. Brinkman, A.H.W. Aten Jr., Int. J. Appl. Radiat. Isot. 14, 503 (1963)

21. I.J. Kim, C.S. Park, H.D. Choi, Appl. Radiat. Isot. 58, 227 (2003)

22. A. Georgiev, IEEE Trans. Nucl. Sci. 40, 770 (1993)

23. I.H. Lazarus et al., IEEE Trans. Nucl. Sci. 48, 567 (2001)

24. C.M. Petrache et al., Nucl. Phys. A 635, 361 (1998)

25. J. Uusitalo et al., to be published

\section{References}

1. M. Leino et al., Nucl. Instrum. Methods B 99, 653 (1995)

2. J. Uusitalo et al., Acta Phys. Pol. B 50, 319 (2019)

3. J. Sarén et al., Nucl. Instrum. Methods B 266, 4196 (2008)

4. J. Hilton et al., Phys. Rev. C 100, 014305 (2019) 